\title{
Study of Correlation Between Platelet Count and Serological Markers of Dengue Infection with Importance of NS1 Antigen in Western Region of India
}

KRUNAL D. MEHTA, BINDIYA GHEDIYA, SHRUTI SHETH, SURAJ KHANDHEDIYA, HITESH SHINGALA, MALA SINHA

\section{ABSTRACT}

Introduction: Dengue virus infection has emerged as a notable public health problem in recent decades in terms of the mortality and morbidity associated with it. Dengue has become endemic in India with outbreaks occurring almost every year.

Aim: This study is to correlate the clinical marker like platelet count and serological markers like lgM and NS1 in the acute stage of dengue infection

Materials and Methods: This was a cross-sectional study of conducted in the Government Hospital, Jamnagar, India, the primary health care centres and the community health care centres, which were covered under the district Jamnagar, all clinically suspected cases of dengue, reported to various outpatient departments, emergency services and admitted patients in our Hospital, were included for this study, between the period of September 2013 to December 2013 over duration of 4 months. Samples from clinically suspected dengue cases were collected and tested for
NS1 and IgM by ELISA depending on days of fever. Platelet counts were obtained from all positive cases. In all positive cases dengue specific parameters like NS1 and IgM were compared against platelet counts.

Statistical Analysis: The Chi-Square test and statistical software Epi info and MS excel was used to find out the statistical significance of the estimate.

Results: Of 1628 samples tested, 563 were positive for one or more dengue parameters. Of the 563, 363 were positive for NS1 only, 200 were positive for IgM only, Thrombocytopenia was more consistently associated whenever IgM was detected compared to NS1 detection.

Conclusion: Inclusion of NS1 in the diagnosis of dengue increases the early diagnosis so as to avoid complications significantly. In confirmed dengue cases with fever, thrombocytopenia is more consistently found whenever IgM is positive compared to NS1 positive and can be used as predictor to reduce the morbidity and moratlity of dengue disease.

\section{INTRODUCTION}

Dengue fever is an acute febrile arbo-viral disease affecting the tropical and subtropical regions of the world. The incidence of this disease has increased over the last 50 years with 2.5 billion people living in areas where dengue is endemic [1]. Severe and potentially lethal type of dengue hemorrhagic fever (DHF) and dengue shock syndrome (DSS) are found more in children now-a-days increasing disease burden [2]. DHF with mortality in most countries is $5 \%$, primarily among young children and adults [3]. Rapid and sensitive laboratory methods required for early detection of the disease to reduce the morbidity and mortality [4]. More specific methods like virus isolation, genomic RNA detection by PCR, antigen and antibody detection by ELISA are available but it needs well trained staff and an expensive setup which is not feasible in peripheral hospital settings [5].

In most cases antibody (lgG/M) detection are commonly used for diagnosis of dengue infection, but time required for appearance of IgM antibody is approximately 4-6 days [6]. Dengue non-structural 1 antigen (NS1) is highly conserved glycoprotein produced in both membrane associated and secretory forms is used as a new biomarker for early diagnosis of dengue infection [7]. NS1 antigen detected by ELISA is present in high concentrations in sera of dengue virus infected patients during early clinical phase of disease [8]. Apart from dengue specific parameters, platelet count is 
the only laboratory test available and easily performed roughly by microscopy without any expensive setup in the peripheral areas and remote area that can support the diagnosis of DHF or DSS [9]. A drop in platelet count below 1,00,000 per $\mathrm{mm}^{3}$ is usually found between the third and eighth day of illness so thrombocytopenia and haemo-concentration are used to detect and monitor DHF [6].

Because of limited available resources in healthcare system in the peripheral areas, we tried to correlate the platelet counts and serological tests for NS1 antigen and antibody detection for early diagnosis of dengue fever.

\section{MATERIALS AND METHODS}

This was a cross sectional study conducted in the Government Hospital, Jamnagar, India, the primary health care centres and the community health care centres, which were covered under the district Jamnagar. The Clinically suspected cases of dengue virus infection coming to the various outpatient departments, emergency services and admitted patients in our Hospital, were included for this study, during the period of September 2013 to December 2013.

Ethical Considerations: This was a analysis of routine laboratory diagnostic work thus ethical approval was not necessary.

\section{Inclusion and Exclusion Criteria}

Clinically suspected patients experiencing febrile illness consistent with dengue fever with two more of the following manifestations:

$\begin{array}{ll}- & \text { Headache } \\ \text { - } & \text { Muscle pain } \\ \text { - } & \text { Hetro-orbital pain } \\ \text { - } & \text { Rash }\end{array}$

A total number of 1628 acute phase blood samples were collected from children and adults age ranging from 0 to 80 year. WHO criteria were followed for inclusion or exclusion of cases of dengue infection and their categorization as DF/ DHF [9]. Total 1225 Cases received within first 5 days of onset of symptoms were tested for NS-1 only, while 403 cases received after 5 days were tested for IgM only.

\section{Data Collection}

A specially designed, semi-structured questionnaire form was used to collect the data and the results of the investigations. Approximately $5 \mathrm{ml}$ of blood samples were collected from all the suspects as a part of the routine laboratory work and the sera were separated and tested for the Dengue NS1 and IgM by ELISA methods.

\section{SEROASSAYS}

\section{NS1 Ag assay}

NS1Ag MICROLISA (J. Mitra \& Co, New Delhi) test kit was used to perform the test. NS1Ag MICROLISA is a solid phase enzyme linked immunosorbent assay (ELISA) based on the "Direct Sandwich" principle. Anti-Dengue NS1 antibodies are coated on Microwells with high reactivity for Dengue NS1 antigen. The samples are added in the wells and then enzyme conjugate (monoclonal anti-dengue NS1 antibodies linked to Horseradish peroxidase (HRPO) added. A sandwich complex is formed in the well wherein dengue NS1 (from serum sample) is "trapped" or "sandwiched" between the antibody and antibody HRPO conjugate. wash buffer is added that will washed off Unbound conjugate. The amount of bound peroxidase is proportional to the concentration of dengue NS1 antigen present in the sample. Upon addition of the substrate buffer and chromogen, a blue colour develops. The intensity of developed blue colour is proportional to the concentration of dengue NS1 antigen in sample. Stop solution is added to limit the enzyme-substrate reaction, and a yellow colour develops which is finally read at 450 $\mathrm{nm}$ spectrophotometrically. Sample results were expressed in terms of ratiowithin 2 hours. As per the manufacturer's guideline, interpretation was done as (i) non reactive for dengue virus NS1 Ag if ratio $<9$, (ii) equivocal for dengue virus NS1 Ag if between 9 to 11, and (iii) reactive for dengue virus NS1 Ag if $>11$ or more was obtained.

\section{Detection of IgM}

All the 403 samples were tested for the presence of dengue specific IgM antibodies by using MAC ELISA, developed and commercialized by NIV (National Institute of Virology), Pune, and recommended by National Vector Borne Disease control programme. Tests were done and results were read as per the literature provided.

\section{Platelet Count}

EDTA blood samples were collected and the platelet count was done by automated analyser and cross checked by light microscopy in the Department of Pathology, G.G.Hospital Jamnagar and interpreted as normal, when the count was between $1500000-450000 / \mathrm{cmm}$ and DHF, when the count was < $1,00,000 / \mathrm{cmm}$ ( WHO cut off for platelet count for DHF).

\section{STATISTICAL ANALYSIS}

The Chi-Square test and statistical software Epi info and MS excel was used to find out the statistical significance of the estimate.

\section{RESULTS}

Out of total 1628 samples tested, 1225 were tested for NS1 and 403 samples were tested for IgM. Out of total 1225 
samples tested for NS1, 909 were males and 316 were females and out of total 403 samples tested for IgM, 259 were males and 144 were females. So out of total 1628 samples tested male predominance $(71.4 \%)$ was there than female.

Out Of total 1628 samples tested a total of 563 (34.6\%) samples were positive for either one or more of the markers (NS1 and IgM) tested.

Of the 563 positive serum samples, 363 (64.5\%) were positive for NS1 only, 200 (35.5\%) were positive for IgM only [Table/Fig-1].

Of the 563 total positive cases of dengue, 393 were male (69.9\%) and 170 were females (30.1\%). Of the 563 total positive cases of dengue, highest positivity was found in age group 11-30 years in both NS1 (65.9\%) and IgM (68\%) positive cases [Table/Fig-2].

Of the 563 total positive cases of dengue, 391 (69.4\%) showed thrombocytopenia. In 363 cases that were positive for NS1, thrombocytopenia was evident in 235 (64.7\%) cases. In contrast, when IgM antibodies were considered for the diagnosis of DI, thrombocytopenia was noted in 148 of 200 (74\%) cases. Association of thrombocytopenia with $\operatorname{lgM}$ was found to be higher. It is also statistically significant ( $p=0.03)$ [Table/Fig-3].

\begin{tabular}{|l|c|c|}
\hline Parameter & Total Positive & Percentage \\
\hline NS-1 Only & 363 & $64.5 \%$ \\
\hline IgM Only & 200 & $35.5 \%$ \\
\hline
\end{tabular}

[Table/Fig-1]: Comparison of efficacy of various dengue specific parameters in the diagnosis of dengue infection.

\begin{tabular}{|c|c|c|}
\hline Age groups (Years) & NS1 Positive & IgM Positive \\
\hline $0-10$ & 26 & 11 \\
\hline $11-20$ & 130 & 64 \\
\hline $21-30$ & 129 & 72 \\
\hline $31-40$ & 50 & 30 \\
\hline $41-50$ & 11 & 11 \\
\hline $51-60$ & 12 & 07 \\
\hline$>60$ & 05 & 05 \\
\hline [Table/Fig-2]: Details of Age groups in positive test results.
\end{tabular}

\begin{tabular}{|c|c|c|c|}
\hline Age groups & $\begin{array}{l}\text { Platelet count } \\
\text { less than } 1 \mathrm{lakh} / \mathrm{ml}\end{array}$ & $\begin{array}{c}\text { Platelet count } \\
\text { more than } 1 \text { lakh } / \mathrm{ml}\end{array}$ & Total \\
\hline NS-1 positive & 235 (64.7\%) & 128 & 363 \\
\hline IgM positive & 148 (74\%) & 52 & 200 \\
\hline Total & 383 & 180 & 563 \\
\hline \multicolumn{4}{|c|}{$\begin{array}{l}\text { [Table/Fig-3]: Comparison of platelet counts with Dengue } \\
\text { parameters. } \\
X^{2}=4.67, d f=1, p=0.03\end{array}$} \\
\hline
\end{tabular}

National Journal of Laboratory Medicine. 2016 Apr, Vol 5(2): 55-59

\section{DISCUSSION}

Since, many decades Dengue is the most important arthropod-borne viral disease of public health significance, the global prevalence of Dengue has grown dramatically in recent decades with estimated 2.5 billion people at a risk of acquiring this viral infection and more than 50 million new Dengue infections being projected annually [10]. Dengue and its severe manifestations: DHF and dengue shock syndrome (DSS), are recognized as important emerging public health problems in tropics and subtropics, thats why it is more important to diagnosis dengue in early period with the help

\begin{tabular}{|l|l|l|}
\hline & $\begin{array}{l}\text { Present } \\
\text { study }\end{array}$ & $\begin{array}{l}\text { Study of } \\
\text { R.D. Kulkarni } \\
\text { et al., [11] }\end{array}$ \\
\hline No. of total cases tested & 1628 & 2104 \\
\hline No. of positive cases & 563 & 320 \\
\hline No. of cases positive for NS-1 only & $363(64.5 \%)$ & $95(30 \%)$ \\
\hline No. of cases positive for IgM only & $200(35.5 \%)$ & $161(50 \%)$ \\
\hline $\begin{array}{l}\text { Thrombocytopenia in total } \\
\text { positive cases }\end{array}$ & $391(68 \%)$ & $220(68.8 \%)$ \\
\hline $\begin{array}{l}\text { Thrombocytopenia in NS-1 } \\
\text { positive cases }\end{array}$ & $235(64.7 \%)$ & $70(73.7 \%)$ \\
\hline $\begin{array}{l}\text { Thrombocytopenia in } \\
\text { lgM positive cases }\end{array}$ & $148(74 \%)$ & $96(59.6 \%)$ \\
\hline $\begin{array}{l}\text { [Table/Fig-4]: Comparison of studies regarding association of } \\
\text { thrombocytopenia with Dengue specific parameters. }\end{array}$ \\
\hline
\end{tabular}

of clinical parameter like platelet counts and serological markers to reduce the morbidity and mortality in dengue.

Detection of dengue specific lgM/lgG is the mainly used for the diagnosis of dengue infection since from long time. The antibodies begin to appear on fifth day of fever in primary infection [11,12]. Sometimes IgM/lgG antibodies cannot be detected before the third day of fever in secondary dengue infection [13]. Therefore, there is some lag period both in primary and secondary dengue when test will give negative results in antibodies specific tests. For early diagnosis of DI the NS1 antigens, is available now a days from day 1 of fever both in primary and secondary infections. When NS1 is positive, there is no need of repeat testing as it is a highly specific marker of Dengue infection [11,12]. This supports the fact that a large number of cases would be missed if NS1 is not included in the test panel [11]. The results of various dengue specific parameters are shown in [Table/Fig-1]. Of the 563 cases which were tested for NS1 antigen only, 363 were positive only for NS1 antigen. It stated that $64.5 \%$ cases were diagnosed early preventing severe complication if we had not included NS1 antigen in the testing panel.

Earlier IgM ELISA and IgG ELISA were widely used diagnostic method for dengue fever in routine laboratory practice. Among the two antibodies, IgG is less reliable marker in the 
diagnosis of dengue infection as both clinical and subclinical infection can produce lgG which may persist for several years affecting the interpretation of testing results. IgG levels could be higher in endemic areas due to continues biting from mosquitoes so, dengue specific lgM is very good marker for acute cases and it may also give idea about secondary dengue infection [12]. In the endemic areas, dengue infection is mainly confirmed by rising titre in paired serology. However, repeat testing for the same infection when first sample was negative or for confirmation of dengue infection is almost not possible in routine clinical practise. When NS1 antigen gives positive results in first four days of illness, there is no need of repeat testing as NS1 is highly specific marker for the diagnosis of dengue infection [12].

In study of R.D. Kulkarni et al.,[11], total 2104 samples were tested, out of which 320 were positive for one or more dengue parameters. Of the total 320 positive cases, 95(30\%) were positive for NS-1 only, 161(50\%) were positive for lgM only [Table/Fig-4].

This study also tried to find the association of dengue parameter with thrombocytopenia.

Of the 563 total positive cases, 383 (68\%) showed thrombocytopenia. In 363 cases that were positive for NS1, thrombocytopenia was evident in 235 (64.7\%) cases. In contrast, when IgM antibodies were considered for the diagnosis of DI, thrombocytopenia was noted in 148 of $200(74 \%)$ cases. Association of thrombocytopenia with IgM was found to be higher. In contrast to study of R.D. Kulkarni et al., [11], and Saroj et al., [13] association of thrombocytopenia with NS-1 was found to be higher that is (73.7\%) and (71.4\%).

Higher association of IgM and thrombocytopenia in our study may be due to most of the cases coming to our hospital were referred from various places. This may result in delay in diagnosis and thus increasing morbidity.

As the NS1 antigen is earliest marker detectable in blood from day one after onset of fever, its assay is an effective tool for early diagnosis so as to avoid complications of dengue infection. Out of these dengue specific parameters, platelet count is the only laboratory parameter performed in remote areas because of cost effectiveness and easy to perform without requiring costly setup, that can support the diagnosis of dengue infection. Such predictions will help to decrease complication due to late treatment and initiate the preventive and control measures well in time for the containment of spread of the disease. Therefore, studies like this will contribute significantly to the clinical management and can reduce morbidity and mortality in dengue infection.

\section{CONCLUSION}

The study draws attention toward the male, young adult age group. Inclusion of NS1 in the diagnosis of dengue increases the early diagnosis so as to avoid complications significantly. In cases of fever, thrombocytopenia is more consistently found in dengue positive subjects and whenever $\operatorname{lgM}$ is detected as compared to NS1 and can be used as an indicator to reduce the complication of dengue disease. The results of this study indicate that to prevent complication in dengue infection and early diagnosis more programmed study with correlation of other important clinical parameter and serological marker is needed in larger study group.

\section{ACKNOWLEDGEMENT}

Authors thank all the G.G. Civil Hospital, Jamnagar Clinic staff for providing support for recruitment of patients and collection of samples, and also acknowledge the Department of Microbiology, Shri M.P. Shah Government Medical College, Jamnagar, for extending support for the study.

\section{REFERENCES}

[1] Smith AW, Chen LH, Massad E, Wilson ME. Threat of dengue to blood safety in dengue-endemic countries. Emerg Infect Dis. 2009;15:8-11.

[2] Whitehead SS, Blaney JE, Durbin AP, Murphy BR. Prospects for dengue virus vaccine. Nat Rev Microbiol. 2007;5:518-28.

[3] World Health Organisation. Key Issues in dengue vector control towards the opererationalisation of a global strategy: report of consultation. Geneva: WHO;1995.(CTD/FIL(DEN)/IC.96.1).

[4] Datta, S. Wattal. C. Dengue NS1 antigen detection: A useful tool in early diagnosis of dengue virus infection. Indian J Med Microbiol. 2010; 28:107-10.

[5] Kassim FM, Izati MN, Apandi YM, Saat Z et al. Use of dengue NS1 antigen for early diagnosis of dengue virus infection. Southeast Asian J Trop Med Public Health. 2011; 42(3):56269.

[6] World Health Organization. 1997. Dengue haemorrhagic fever: Diagnosis, treatment, prevention and control. $2^{\text {nd }}$ edition. Geneva, Switzerland. Chapter 4, Laboratory Diagnosis.

[7] Subedi D and Taylor-Robinson AW. Laboratory diagnosis of dengue infection: current techniques and future strategies. Open Journal of Clinical Diagnostics. 2014; 4: 63-70.

[8] Kumarasamy V, Wahab AHA, Chua SK, Hassan Z et al. Evaluation of a commercial dengue NS1 antigen-capture ELISA for laboratory diagnosis of acute dengue virus infection. J Virol. Methods. 2007; 140:75-79.

[9] World Health Organization. Dengue haemorrhagic fever: Diagnosis, treatment, prevention and control. $2^{\text {nd }}$ edition. Geneva, Switzerland: Chapter 2, Clinical Diagnosis, 1997. p. 12-23.

[10] Chakravarti A, Matlani M, Kashyap B, Kumar A. Awareness of changing trends in epidemiology of dengue fever is essential for epidemiological surveillance. Indian J Med Microbiol. 2012;30:222-26.

[11] Kulkarni RD, Patil SS, Ajantha GS, Upadhya AK, Kalabhavi AS, Subhada RM et al. Association of platelet count and serological markers of dengue infection - importance of NS1 antigen. Indian Journal of Medical Microbiology. 2011; 29(4): 359-62. 
[12] Peeling RW, Artsob H, Pelegrino JL, Buchy P, Cardosa MJ, Devi S, et al. Evaluation of diagnostic tests: Dengue. Nat Rev Microbiol. 2010;8: S30-37.

[13] Golia S, Hittinahalli V, Sujatha K, Reddy M, Kamath AS. Serodiagnosis of Dengue using NS1 antigen, dengue IgM, dengue $\lg G$ antibody with correlation of platelet count. International journal of $A \mathrm{~J}$ institute of medical sciences. 2012 ;1(2):112-17.

\section{AUTHOR(S):}

1. Dr. Krunal D. Mehta

2. Dr. Bindiya Ghediya

3. Dr. Shruti Sheth

4. Dr. Suraj Khandhediya

5. Dr. Hitesh Shingala

6. Dr. Mala Sinha

\section{PARTICULARS OF CONTRIBUTORS:}

1. Assistant Professor, Department of Microbiology M.P. Shah Medical College, Jamnagar, Gujarat, India.

2. Resident, Department of Microbiology, M.P. Shah Medical College, Jamnagar, Gujarat, India.

3. Tutor, M.D. Microbiology, GCS medical college, Hospital \& Research Centre, Ahmedabad, Gujarat, India.

4. Tutor, PSM Department, GMERS Medical College, Dharpur, Patan, Gujarat, India.
5. Associate Professor, Department of Microbiology, M.P. Shah Medical College, Jamnagar, Gujarat, India.

6. Professor and Head, Department of Microbiology, M.P. Shah Medical College, Jamnagar, Gujarat, India.

\section{NAME, ADDRESS, E-MAIL ID OF THE CORRESPONDING AUTHOR:}

Dr. Krunal D. Mehta,

Assistant Profesor, Department of Microbiology, Near Mental Hospital, VIkas Gruh Road, Patel Colony, Jamnagar-361008, Gujarat, India.

E-mail: Krunaldmehta@yahoo.co.in

\section{FINANCIAL OR OTHER COMPETING INTERESTS:} None. 